\title{
Uma abordagem de segmentação de objetos em nuvem de pontos para FOD
}

\author{
Filipe Santiago de Queiroz* \\ Carlos Henrique Quartucci Forster ${ }^{* *}$ \\ * Divisão de Ciência da Computação, ITA, SP, (e-mail: \\ filipesantiago.qrz@gmail.com) \\ ** Divisão de Ciência da Computação, ITA, SP, (e-mail: \\ forster@ita.com.br)
}

\begin{abstract}
This work presents an approach to point cloud segmentation for Foreign Object Debris (FOD) detection in flight tracks and a proposal for their complete removal process. It is part of the methodology the use of two approaches, one using regions based method and the other using a hybrid method. The term FOD or foreign object is used for objects not belonging to flight lanes and taxiways of airports. The existence of FOD in flight lanes is a recurring problem in aviation in general, which must be kept under constant monitoring. The consequences of this problem range from high aircraft maintenance costs to overhead expenses such as hotel nights due to delayed flights. The approaches proposed here fall under a process of detection and removal of debris. The proposed differential is the use of pre-processing in the raw data, from the capture sensor, to improve the results of the segmentation of objects. In the removal process it is proposed the use of a robot integrated with the detection systems already present on the tracks. For validation of the methods, a comparative analysis between some of the main works in this area will be presented, as well as tests in a set of data for demonstration of the results.
\end{abstract}

Resumo: Este trabalho apresenta uma abordagem para segmentação de nuvem de pontos para detecção de FOD (Foreign Objects Debris) em pistas de voo e uma proposta para o processo completo de remoção. Faz parte da metodologia de segmentação a utilização de duas abordagens, uma com método baseado em regiões e a outra com um método híbrido. O termo FOD ou objeto estranho é utilizado para objetos intrusos às pistas de voo e taxiamento dos aeroportos. A existência de $F O D$ em pistas de voo é um problema recorrente na aviação de forma geral e que precisa estar sempre em monitoramento. As consequências deste problema vão desde altos valores gastos com manutenção dos aviões até com despesas indiretas como diárias em hotéis devido ao atraso de voos. As abordagens propostas aqui se enquadram em um processo de detecção e remoção dos FOD's. O diferencial proposto é a utilização de pré-processamentos nos dados brutos, oriundos do sensor de captura, para melhoria nos resultados da segmentação dos objetos. No processo de remoção é proposta a utilização de um robô integrado aos sistemas de detecção já presentes nas pistas e que faz uso do processo de detecção citado. Para validação dos métodos, uma análise comparativa entre alguns dos principais trabalhos nesta área serão apresentados, assim como testes em um conjunto de dados para demonstração dos resultados.

Keywords: Point Cloud; Segmentation Process; Computer Vision; Robotics; FOD.

Palavras-chaves: Nuvem de Pontos; Processo de Segmentação; Visão Computacional; Robótica; FOD.

\section{INTRODUÇÃO}

Diariamente o processo de recolhimento de FOD (Foreign Objects Debris) ou detritos em pistas de pouso e decolagem ao redor do mundo é executado exaustivamente. A procura por FOD's espalhados ao longo destas áreas fazem parte da rotina de qualquer aeroporto. Tal preocupação se deve ao fato de que FOD's podem ser prejudiciais de diversas maneiras. Dentre os principais prejuínzos podemos destacar o elevado valor utilizado para manutenção em aeronaves danificadas e acidentes envolvendo pessoas.
Segundo a Administração de Aviação Federal dos Estados Unidos (Federal Aviation Administration - FAA) FAA (2013), FOD é qualquer objeto estranho que não pertença à pista de voo, à pista de taxiamento ou à área da rampa. Neste contexto até mesmo uma pessoa no lugar errado pode ser considerada um detrito. O problema dos FOD's em pistas de voo é tão sério que as principais organizações de aviação civil (ICAO, FAA, EUROCONTROL e ANAC) e militar do mundo (U.S. Air Force, FAB) estão sempre a promover melhorias nos mecanismos de limpeza e manutenção das aerovias, além de incentivar a pesquisa e inovação em sistemas de detecção e remoção de FOD's. 
Estima-se que os danos resultantes custem à indústria aeroespacial a quantia de 4 bilhões de dólares por ano. Os custos indiretos tendem a ser 10 vezes maiores que os custos diretos (Boeing). A Boeing em seu relatório não descreve detalhadamente como chegou nos valores mencionados mas estudos mostram que tal número é possível de ser alcançado se levarmos em consideração o número de aviões, aeroportos e voos que acontecem diariamente e que estão espalhados ao redor do globo.

O cenário almejado, consiste na utilização dos equipamentos já presentes nos aeroportos, como os radares microondas, trabalhando em conjunto com o sistema de remoção de objetos. Quando o radar detecta o FOD, essa informação é enviada para uma central de detecção, na qual é responsável por gerenciar a atuação dos robôs nas pistas de voos, observando a melhor disponibilidade para enviarlhes. Uma vez concedida a permissão para o robô navegar, ele se desloca até o local do detrito, utilizando todo a aparelhagem para navegação autônoma. Inicia-se então o processo de detecção e remoção de objetos com o auxilio do braço mecânico. Ao finalizar a remoção, o robô volta para sua estação base (Figura 1).

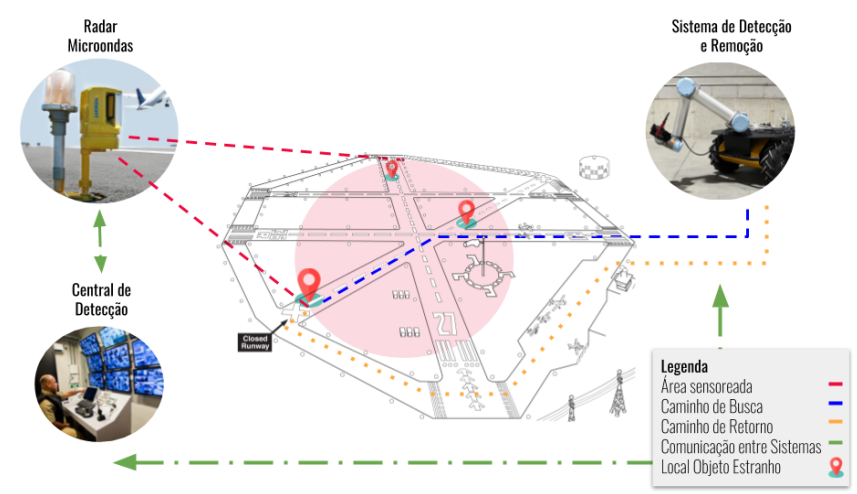

Figura 1. Cenário ideal de atuação, na qual o processo de detecção de objetos estranhos estaria inserido.

Visualizando tal cenário colaborativo onde robôs e sensores micro-ondas atuam para detecção e remoção de FOD's de pista de voo, o objetivo do presente trabalho é propor sistemas robustos capazes de detectar FOD's utilizando segmentação de nuvem de pontos, servindo como préprocessamento para manipulação robótica, a partir do uso de sensores de profundidade de baixo custo acoplados em um robô do tipo rover. O sistema se concentra na segmentação, ou seja, na identificação dos objetos estranhos utilizando nuvens de pontos. Quanto aos demais aspectos, relacionados à manipulação robótica, embora relevantes, não pertencem ao escopo deste trabalho.

\section{REVISÃO BIBLIOGRÁFICA}

O reconhecimento de objetos através de nuvem de pontos 3D é um problema desafiador e que têm sido explorado cada vez mais, especialmente com o desenvolvimento de sensores em profundidade como o Kinect, que permitem a geração de uma grande quantidade de dados para aproximação do modelo de um objeto. Zrira et al. foca no problema de reconhecimento de objetos a partir de uma nuvem de pontos. Seu objeto de estudo é saber o que é aquele objeto e não apenas segregá-lo em uma cena. Para isso utilizou-se dos principais algoritmos de extração de descritores que a ferramenta Point Cloud Library(PCL) Rusu and Cousins (2011) dispõe para comparar seu desempenho com seu algoritmo o proposto.

Quando nos deparamos com um ambiente onde os objetos estão dispostos de forma desordenada e suas formas são arbitrárias, agarrar objetos na desordem é significativamente mais difícil do que agarrar objetos isoladamente. Boularias et al. propõe uma solução para este problema implementando um algoritmo que utiliza probabilidades de transição estocástica entre os estados observados.A função de transição aprendida é utilizada apenas para recalcular os valores das ações executadas nos estados observados com diferentes políticas. Valores de novos estados são obtidos pela regressão dos valores das ações. Por fim, aplica-se um agrupamento espectral para detecção dos diferentes objetos na imagem.

Kim and Sukhatme atentam para a importância da noção de espaço que o robô precisa ter em um ambiente para sua locomoção. Deste modo propõem uma abordagem de rotulação semântica, classificando por exemplo, como "empurrável" ou "levantável", os objetos dispostos no ambiente. Para isso, utiliza-se de técnicas para extração de formas geométricas das nuvens de pontos junto com o classificador semântico proposto. Juntamente com o classificador, foi aperfeiçoado o processo de segmentação dos objetos com o objetivo de reduzir as incertezas de manipulação. $\mathrm{O}$ novo recurso é a rotulagem semântica que pode ser aplicada diretamente ao planejamento de manipulação.

Tabela 1. Resumo das principais referências bibliográficas.

\begin{tabular}{|c|c|c|c|}
\hline Aspectos & Autor & Sensores & Abordagem \\
\hline \multirow{4}{*}{$\begin{array}{l}\text { Detecçāo de } \\
\text { Detritas }\end{array}$} & $\begin{array}{c}\text { Öztürk e } \\
\text { Kuzucuoğlu (2016) }\end{array}$ & $\begin{array}{l}\text { Sensores } \\
\text { Micro-ondas }\end{array}$ & $\begin{array}{l}\text { Coordenação entre robôs para } \\
\text { limpeza das pistas. }\end{array}$ \\
\hline & Cao et al. (2016) & Imagem Visível & $\begin{array}{l}\text { Aplicação de rede neurais para } \\
\text { detecção de detritos . }\end{array}$ \\
\hline & $\begin{array}{l}\text { Dabbiru et al. } \\
\quad(2015)\end{array}$ & $\begin{array}{c}\text { Imagens de } \\
\text { Satélites e LIDAR }\end{array}$ & $\begin{array}{c}\text { Aplicação de correlação entre os } \\
\text { sensores. }\end{array}$ \\
\hline & Han et al. (2013) & Imagem Visível & $\begin{array}{l}\text { Classificação de detritos usando } \\
\text { SIFT e SVM. }\end{array}$ \\
\hline \multirow{4}{*}{$\begin{array}{l}\text { Manipulação } \\
\text { Robótica }\end{array}$} & $\begin{array}{l}\text { Jain e Argall } \\
\quad(2016)\end{array}$ & $\begin{array}{l}\text { Kinect RGB-D e } \\
\text { Braço Robótico }\end{array}$ & $\begin{array}{c}\text { Estuda o melhor ponto para o } \\
\text { Grasping. }\end{array}$ \\
\hline & Zhu (2016) & Kinect RGB-D & $\begin{array}{l}\text { Aplicação de segmentação semântica } \\
\text { separando em } 4 \text { Grupos (Objetos, } \\
\text { Paredes, Piso e Coisas) }\end{array}$ \\
\hline & $\begin{array}{l}\text { Saxena et al. } \\
\quad(2008)\end{array}$ & $\begin{array}{l}\text { Imagem Visível e } \\
\text { Braço Robótico }\end{array}$ & $\begin{array}{l}\text { Utiliza-se de várias imagens do } \\
\text { mesmo objeto e estima qual melhor } \\
\text { ponto para o Grasping. }\end{array}$ \\
\hline & $\begin{array}{c}\text { Mojtahedzadeh } \\
\quad(2016)\end{array}$ & LIDAR's & $\begin{array}{c}\text { Estuda a forma dos objetos e planeja } \\
\text { a melhor sequência para remoção } \\
\text { utilizando um modelo de estimaçâo } \\
\text { de posturas. }\end{array}$ \\
\hline \multirow{3}{*}{$\begin{array}{l}\text { Segmentação de } \\
\text { Nuvem de } \\
\text { Pontos }\end{array}$} & Zrira et al. (2017) & Kinect $R G B-D$ & $\begin{array}{c}\text { Aplica extração de feiçōes de objetos } \\
\text { para depois classificá-los. }\end{array}$ \\
\hline & $\begin{array}{c}\text { Boularias et al. } \\
\quad(2015)\end{array}$ & $\begin{array}{l}\text { Kinect } R G B-D \text { e } \\
\text { Braço Robótico }\end{array}$ & $\begin{array}{l}\text { Aplicação de Spectral Clustering e } \\
\text { aprendizado por Reforço. }\end{array}$ \\
\hline & $\begin{array}{c}\text { Kim e Sukhatme } \\
\text { (2014) }\end{array}$ & Kinect $R G B-D$ & $\begin{array}{l}\text { Extrai informações sobre a forma do } \\
\text { objeto para indexá-los. }\end{array}$ \\
\hline
\end{tabular}

\section{SEGMENTAÇÃO DE NUVEM DE PONTOS}

Como solução, neste trabalho são propostos dois processos de segmentação. O Processo I utiliza as informações de distância entre os pontos mais um método baseado em 
regiões. O Processo II, também utiliza métodos baseados em regiões mas com a adição da informação de cor disponibilizada pelo sensor de aquisição dados.

Os detritos ou objetos estranhos apresentam algumas características importantes que precisam ser levadas em consideração no processo de segmentação.

\subsection{A forma do objeto estranho}

A principal observação a ser feita está relacionada com sua geometria. O que levou este trabalho a adotar uma abordagem por nuvem de pontos e não a utilização de imagens foi a forma dos detritos. Os detritos como vimos nos capítulos anteriores, apresentam um formato geométrico aleatório, deste modo fica difícil a construção de um modelo fixo baseado na geometria padrão. Segundo o U.S. Department of Transportation UDT (2010) os principais objetos encontrados podem ser: peças dos motores de aviões, ferramentas mecânicas, material das pistas de voo e taxiamento(asfalto ou ladrilhos soltos), materiais plásticos, objetos de uso pessoal como lixas, cortadores de unha, canetas e lápis, ou seja uma infinidade de objetos.

Neste caso, o robô precisa ter a capacidade de detectar objetos que estejam acima do solo, não se importando nesse primeiro momento com qual tipo de objeto ele estaria manipulando. Os sensores de profundidade são muito úteis nesse aspecto pois nos devolvem um mapeamento 3D do ambiente possibilitando a detecção dos objetos e a partir desse momento utilizar métodos de segmentação que consigam separar os detritos.

\subsection{Processo I: Segmentação utilizando método baseado em regiões}

Para contornar as caraterísticas dos detritos, uma das abordagens utilizadas foi a elaboração de um processo de segmentação que adiciona passos de pré-processamento seguido por um método baseado em regiões para segregar os objetos estranhos. Tal processo pode ser visto na Figura 2 e será descrito em detalhes.
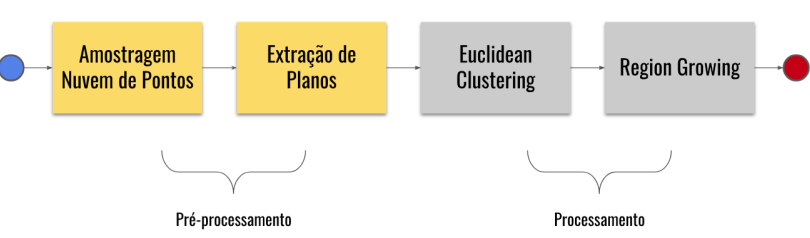

Figura 2. Processo de segmentação utilizando extração de planos como pré-processamento e o algoritmo Region Growing.

\begin{abstract}
Amostragem de Nuvem de pontos Uma extração bruta da nuvem pontos fornecida pelo sensor contém mais 300.000 pontos. Para melhorar o desempenho do processamento dos pontos, foi utilizada uma amostragem destes dados com filtro disponibilizado pela biblioteca $P C L$ para diminuir a resolução de entrada de dados. O filtro funciona como compressor de pontos. Ele cria um 3D voxel grid que contém um conjunto de minusculas caixas 3D no espaço. Logo em seguida para cada voxel, todos os pontos presentes são aproximados de acordo com seu centroide.
\end{abstract}

É um processo lento mas que apresenta bons resultados para extração dos pontos pertencentes ao plano de uma superfície.

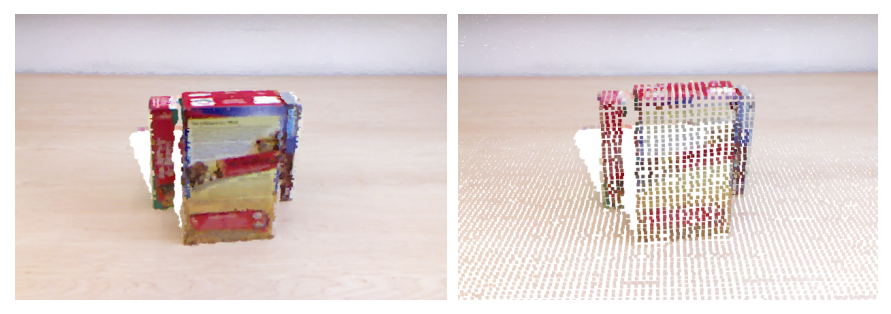

Figura 3. Exemplos de redução de quantidade de pontos.

Extração de Planos Feita a redução de pontos, o próximo passo é a extração do plano que representa a superfície onde o objeto está disposto. A função pcl::SACSegmentation usa o método RANSAC (Fischler and Bolles (1981)) para encontrar todos os pontos dentro de uma nuvem de pontos que suportam um modelo de plano. Isso retorna os quatro coeficientes planares e todos os pontos internos no maior componente planar da nuvem. Usando esses índices de pontos, o pcl::ExtractIndices pode projetar as inliers no modelo de plano e criar uma nova nuvem de pontos, que representa os objetos na superfície.

Para remover pontos indesejados que estão localizados no plano, mas fora da superfície, um filtro pcl::PassThrough é utilizado. Este filtro permite que os pontos da nuvem passem para uma nuvem de saída, dado que os pontos satisfazem um intervalo especificado ao longo de uma determinada dimensão. Neste trabalho foi aplicado o filtro nos três eixos (x, y e z), para segregar uma área específica (Figura 4).
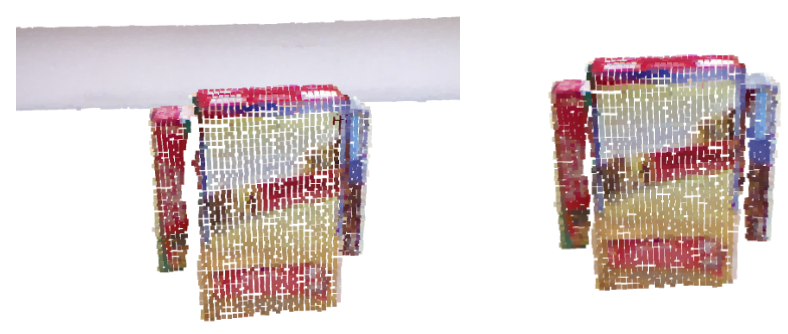

Figura 4. Resultado da remoção da superfície planar.

Euclidean Clustering Com os índices dos pontos sob o plano em mãos, é aplicado um agrupamento de pontos através da distância euclidiana entre os mesmos. Define-se então qual a distância mínima necessária entre pontos que o definem como integrante ou não daquele agrupamento. Para tal tarefa foi utilizado a função pcl::EuclideanClusterExtraction da biblioteca $P C L$, que fornece ferramentas para clusterização. Tal método aplica uma abordagem simples de agrupamento de dados, utilizando a distância Euclidiana, que pode ser implementada subdividindo o espaço usando caixas de largura fixa ou uma estrutura de dados chamada Kd-tree (Figura 5). Esta representação particular é muito rápida de construir e é útil para situações em que seja necessária uma representação volumétrica do espaço ocupado.

Esse é um passo muito importante no processo pois realiza a primeira "quebra de objetos". Ele funciona bem para 

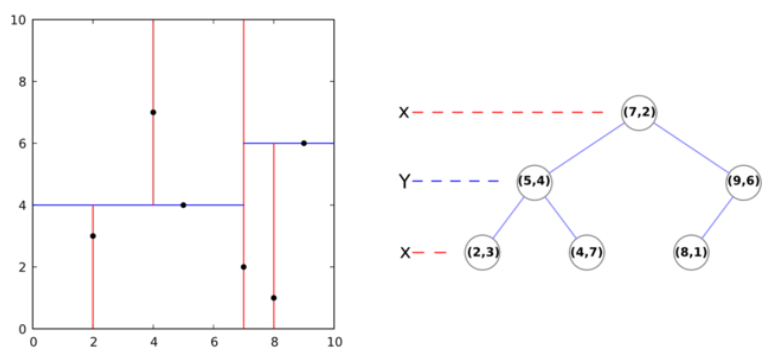

Figura 5. Estrutura de dados Kd-Tree.

objetos que não estão sobrepostos ou seja estão separados e ao final deste passo temos uma lista de agrupamento de pontos.
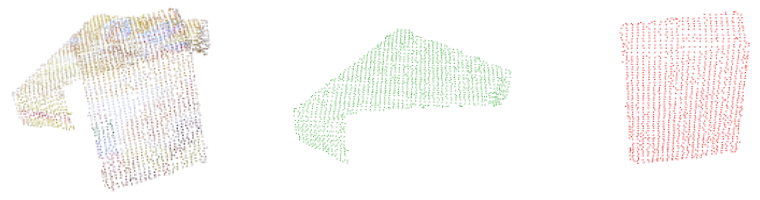

Figura 6. Resultado do processo de agrupamento.

Region Growing No final deste processo se encontra o método baseado em regiões. Essa abordagem de segmentação examina pontos vizinhos aos pontos da semente inicial e determina se os vizinhos destes pontos devem ser adicionados à região. Esta etapa foi adicionada na parte final do processo, pois utiliza outras informações que suplementam o processo anterior.

No Region Growing é possível adicionar limites sobre ângulos e curvatura. A primeira propriedade define o ângulo em radianos que será usado como o intervalo permitido para o desvio normal dos pontos. Se o desvio entre as normais dos pontos for menor do que o limiar de suavidade, sugerese que eles estejam no mesmo agrupamento. A segunda propriedade é responsável pelo limiar de curvatura. Se dois pontos tiverem um pequeno desvio normal, a disparidade entre suas curvaturas será testada, e se esse valor for menor que o limite de curvatura, o algoritmo continuará o crescimento do agrupamento usando o novo ponto adicionado.
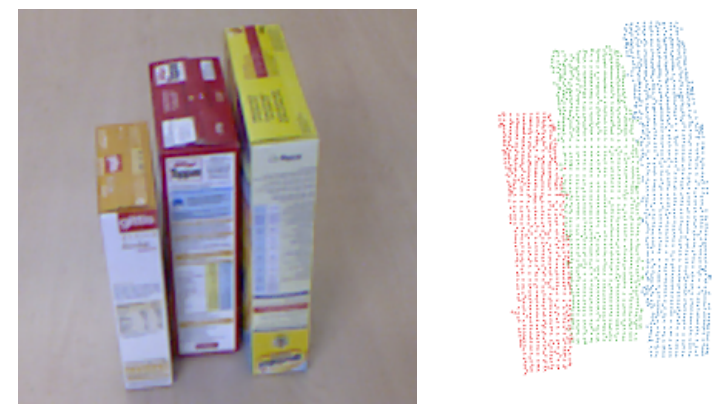

Figura 7. Resultado final, após aplicação do processo baseado em regiões.

\subsection{Processo II: Segmentação utilizando método híbrido}

Esta segunda abordagem utiliza na parte de pré- processamento os mesmos processos da abordagem anterior, adicionando um método baseado em regiões e outro baseado em aprendizado de máquina. Neste caso foi utilizado o algoritmo K-Means para suavização de cores da nuvem de pontos e Region Growing customizado para crescer pela informação de cor (Figura 8).

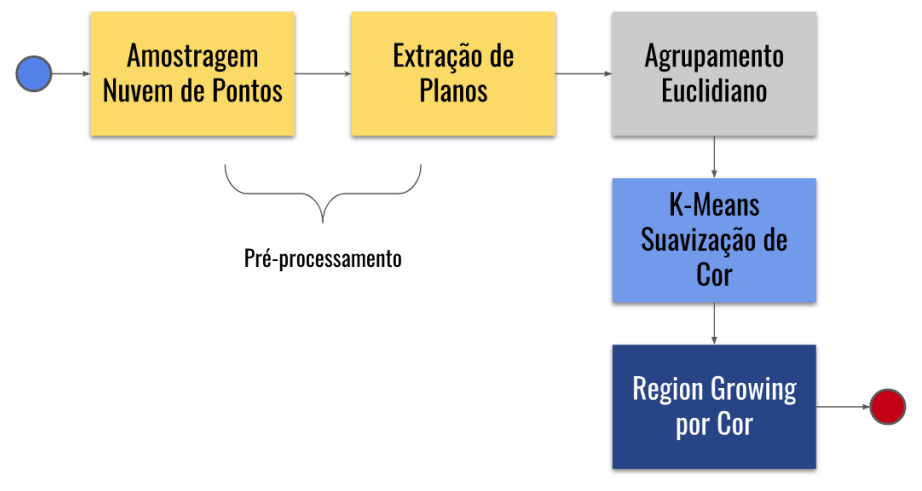

Figura 8. Processo de segmentação híbrido (Euclidean Clustering + K-Means + Region Growing).

Pré-processamento Nesta nova abordagem temos a mesma fase de pré-processamento dos dados da nuvem de pontos. Tal passo é fundamental para o sucesso dos algoritmos subsequentes na tarefa de segmentação. Neste ponto propomos que o Euclidean Clustering se torne parte do pré-processamento dos dados de nuvem de pontos. Visto que sua função inicial de agrupamento de áreas com densidade alta de pontos prepara o terreno para o próximo passo, quebrando o problema em problemas menores.

Suavização com K-Means O sensor de aquisição de dados utilizado neste estudo, prover as informações de profundidade e cor (Red, Green and Blue). A primeira informação vêm sendo utilizada ao longo de todo o corrente trabalho, a construção da nuvem de pontos é proveniente da tal informação. A segunda informação ganha um destaque somente nesta etapa. Utilizando como inspiração a utilização extensiva do algoritmo $K$-Means em processamento de imagens, em diversas aplicações. Neste estudo este método foi incluído com a finalidade de suavizar a informação de cor presente na nuvem de pontos reutilizando a implementação na linguagem $\mathrm{C}++$ fornecida por Castro (2015).

Sabendo que o algoritmo seguinte no processo utilizará um crescimento de região por cor, esta etapa suaviza os possíveis contrastes encontrados nos objetos. Por exemplo, se nos deparamos com uma caixa de um produto qualquer, seu logo contendo fontes coloridas e paisagens no fundo podem influenciar o comportamento do Region Growing por cor. Grandes variações de cor podem afetar a segmentação neste caso específico.

Para atingir tal objetivo, utilizou-se como dados de entrada no K-Means os valores de cor dos pontos. Com o agrupamento resultante do método, foi calculado um valor médio das cores pertencentes àquele agrupamento. Com esse valor médio em mãos, foram atualizados todos os pontos da nuvem de entrada com seu respectivo valor médio, mudando toda a coloração da nuvem inicial.

Region Growing por Cor Na etapa final deste processo, temos o método baseado em regiões Region Growing 

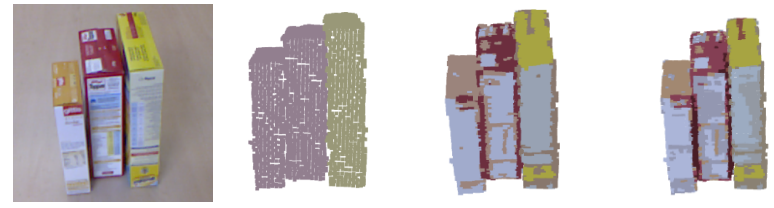

Figura 9. Resultados variando os valores de $\mathrm{K}=\{$ Entrada, $1,3,5\}$.

que desta vez está modificado para fazer o agrupamento observando a informação da coloração dos pontos.

Existem duas diferenças principais entre o algoritmo baseado em cores e o anterior. O primeiro é que agora ele usa cores em vez de normais. A segunda é que ele usa o algoritmo de mesclagem para fazer o controle de sobre/sub segmentação. Após a segmentação, é feita uma tentativa de unificar agrupamentos com cores próximas. Dois aglomerados vizinhos com uma pequena diferença entre a cor média são mesclados. Em seguida, a segunda etapa de mesclagem se inicia. Durante essa etapa, todo agrupamento único é verificado pelo número de pontos que ele contém. Se esse número for menor que o valor definido pelo usuário, o agrupamento atual será mesclado com o agrupamento vizinho mais próximo.
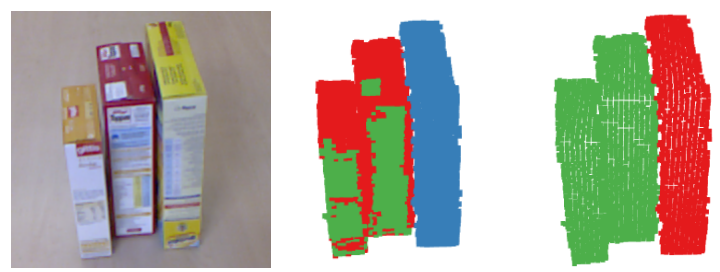

Figura 10. Resultados variando os valores de distância entre cores.

\section{METODOLOGIA DE AVALIAÇÃO}

Esta seção apresenta as métricas e o conjunto de dados utilizados e uma descrição dos experimentos realizados.

\subsection{Dataset}

Vários datasets de segmentação de objetos $R G B-D$ estão disponíveis publicamente. Neste trabalho utilizamos um deles para realização das avaliações de desempenho. Abaixo descrevemos sobre esse dataset e discutimos suas características.

Object Segmentation Database Object Segmentation Database - OSD Andreas (2015) foi gravado pelo grupo Vision for Robotics da Universidade de Tecnologia de Viena. Este dataset contém mais de 100 cenas diferentes capturadas de um único ponto de vista usando uma câmera $R G B-D$. As cenas apresentam vários objetos semelhantes a caixas e objetos cilíndricos que residem em uma mesa.

Todas as nuvens de pontos no conjunto de dados são fornecidas com anotações. Os autores derivaram essas anotações das imagens coloridas das cenas, presumivelmente usando ferramentas automáticas de segmentação. Devido à calibração ligeiramente imprecisa entre os sensores $R G B$ e de profundidade da câmera, os limites dos objetos nas imagens coloridas e de intervalo não coincidem exatamente.
Portanto, os rótulos derivados de imagens coloridas tendem a saltar sobre os limites geométricos dos objetos. Isso é indesejável, uma vez que estamos interessados em avaliar o desempenho de algoritmos de segmentação geométrica. Por esse motivo, retificamos manualmente as anotações para obter rotulagens geometricamente consistentes.

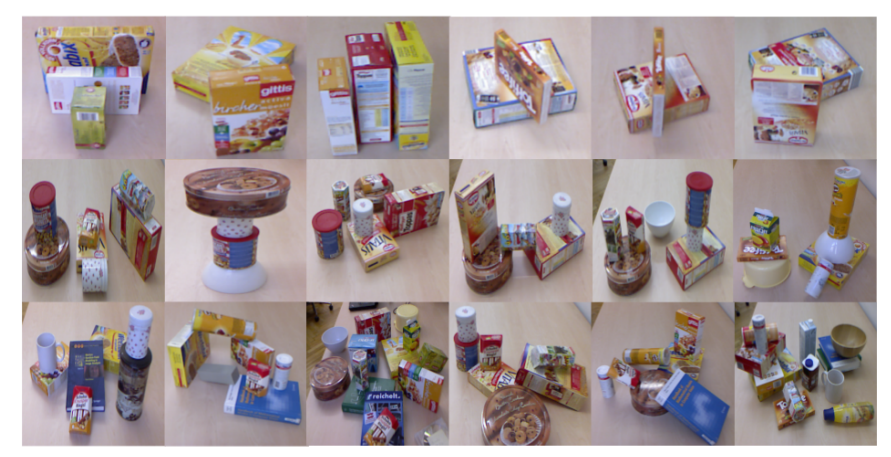

Figura 11. Exemplo de imagens do OSD dataset.

Na maioria das cenas, os objetos obstruem a mesa em que residem. Geralmente, um objeto oculto parece estar dividido em várias partes desconectadas. Os próprios dados da nuvem de pontos não fornecem evidências suficientes de que ambas são partes do mesmo objeto. Portanto, não é razoável esperar que um algoritmo de segmentação lhes atribua o mesmo rótulo, a menos que o conhecimento prévio sobre composição de cena e formas de objeto seja usado. Para não penalizar nosso algoritmo por algo que ele não foi projetado para fazer, alteramos as anotações de verdade do solo para que partes geometricamente desconectadas do mesmo objeto recebam rótulos diferentes.

\subsection{Métricas Utilizadas}

Para medir os resultados da segmentação adotamos uma métrica quantitativa chamada Positive/Negative scores utilizada por Ückermann et al. (2012). As anotações foram feitas manualmente, ou seja, conseguimos dizer se a segmentação foi correta ou não. Caso a segmentação seja parcial tal valor é contabilizado como uma porcentagem de falso negativo ou falso positivo dependendo da cena. Para este fim, consideramos o conjunto de pontos da nuvem de entrada $R_{i}$ e $S_{i}$ o conjunto de pontos da nuvem segmentada. $T P_{i}=R_{i} \cup S_{i}$, denota a sobreposição de ambos conjuntos, isto é, o conjunto de pontos corretamente segmentados (Equação 1).

$$
t p=\frac{1}{n} \sum_{i=1}^{n} \frac{\left|T P_{i}\right|}{\left|R_{i}\right|}
$$

\subsection{Experimentos}

Como experimentos utilizamos a seguinte abordagem. O $O S D$ dataset foi subdivido em três classes, relacionadas a complexidade das cenas. Temos cenas com complexidade baixa, média e alta. Sendo assim os testes obedeceram a seguinte ordem:

- Aplicação do Processo I baseado em regiões para as três classes de complexidade do dataset; 
- Aplicação do Processo II baseado em um método híbrido para as três classes de complexidade do dataset;

- Aplicação dos Processos I e II em duas nuvens de pontos que simulam o ambiente real (ferramentas e detritos);

- Comparação entre os processos propostos e métodos utilizados no Capítulo 4;

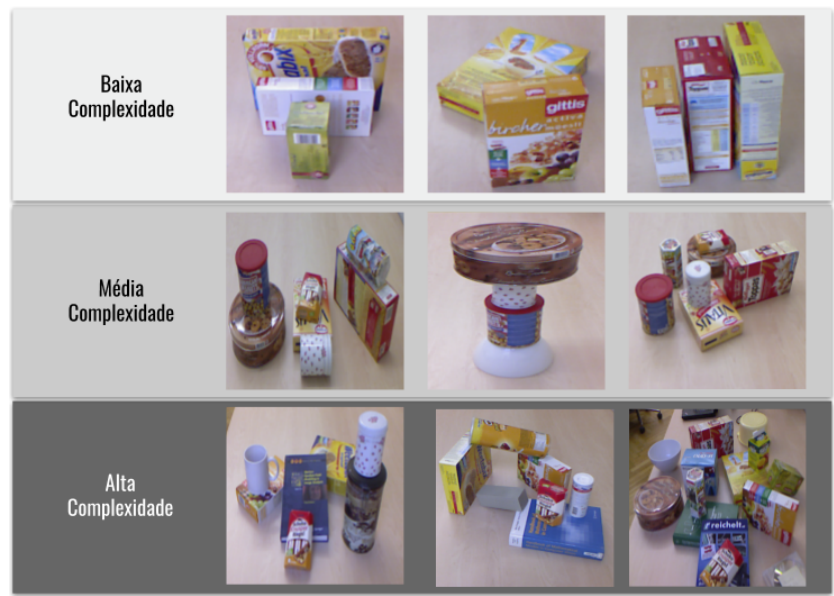

Figura 12. Exemplos de imagens e suas respectivas complexidades.

\section{RESULTADOS}

Abaixo temos os resultados dos processos de segmentação sobre as nuvens de pontos do dataset $O S D$ (Figura $14 \mathrm{e}$ Figura 15). Uma amostragem das nuvens foi selecionada para representar as classes de complexidade do dataset. Por exemplo, na Figura 13 temos o resultado dos processos propostos, para nuvens de pontos categorizadas como baixa complexidade (Tabela 2). Simplificando a notação o Processo I se refere ao processo baseado em regiões e o Processo II ao processo de segmentação híbrido já descritos nos capítulos anteriores.
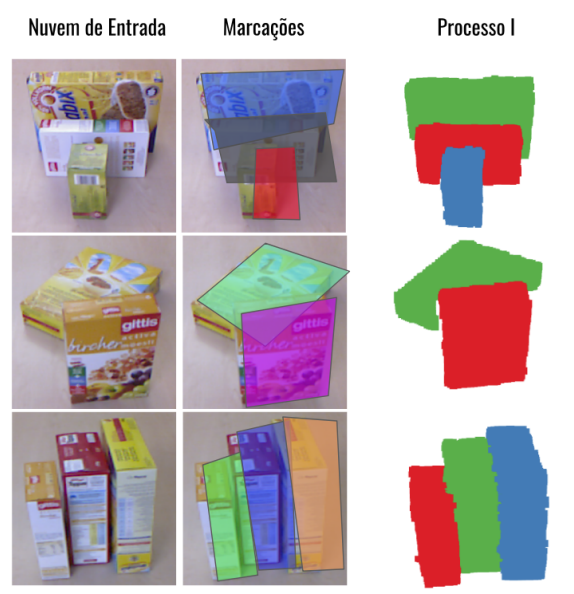

Processo II
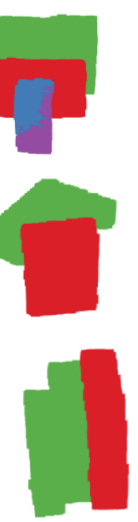

Figura 13. Resultado da segmentação em cenas com baixa complexidade.

\subsection{Discussão}

O desempenho do processo baseado em regiões apresenta os melhores resultados frente aos outros algoritmos. Nossa

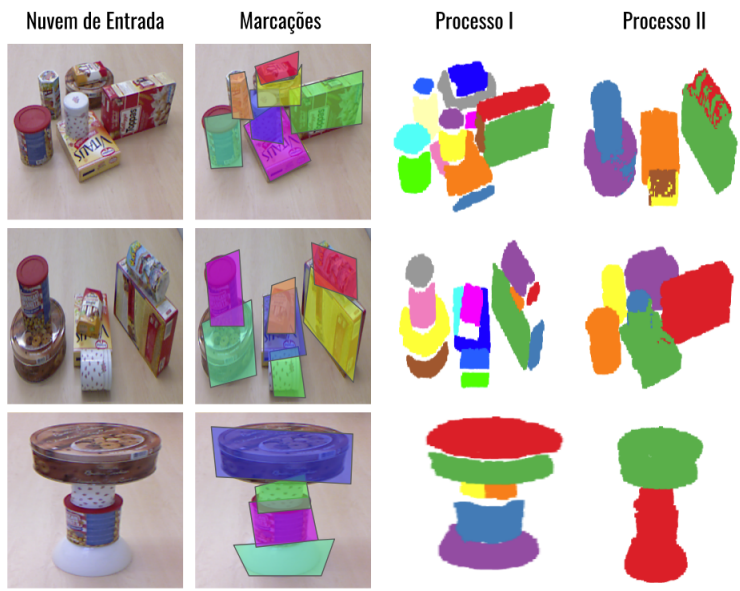

Figura 14. Resultado da segmentação em cenas com média complexidade.
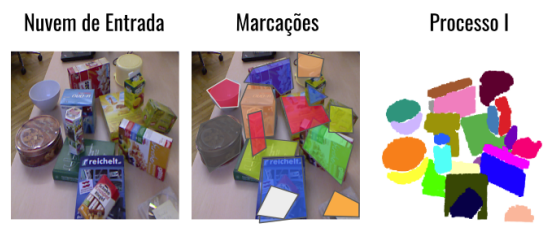

Processo II
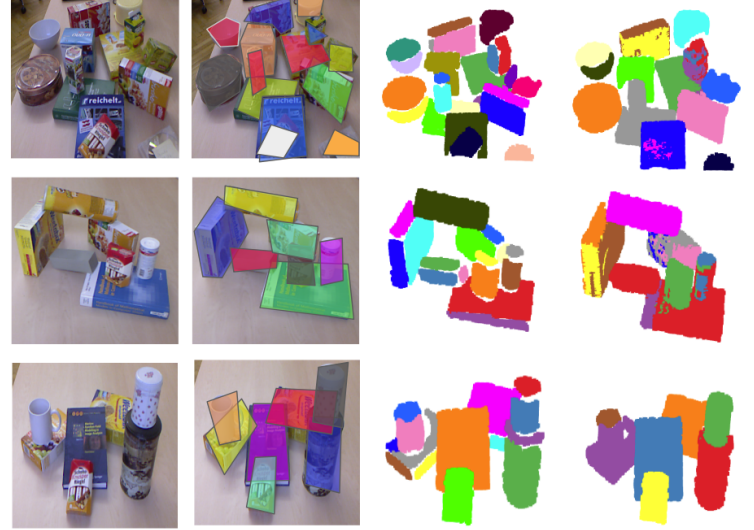

Figura 15. Segmentação em cenas com alta complexidade.

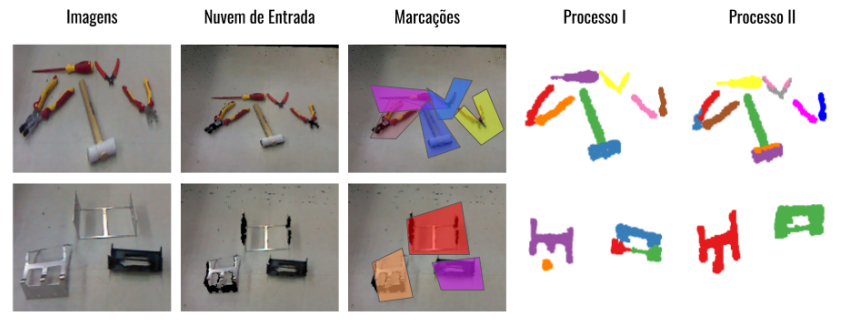

Figura 16. Segmentação em cenas que simulam o ambiente real, contendo ferramentas e detritos. Imagens coletadas do robô Husky.

métrica mede apenas o que o processo acertou ou acertou parcialmente, devido a característica de ter sido rotulado manualmente. Tal métrica nos dá um vislumbre de qual seria a melhor abordagem, mas que serve de impulso para uma outra rodada de testes utilizando outras métricas, como Under/Over segmentation que mostram a relação entre os rótulos verdadeiros e os rótulos que o algoritmo proporcionou. Podemos notar também a influencia dos préprocessamentos utilizados em nossas abordagens. Tanto o Euclidean Clustering como o K-Means precisam que seus parâmetros sejam configurados corretamente, caso contrário teremos efeitos prejudiciais na segmentação. Podemos constatar que a informação dos vetores normais presentes 
Tabela 2. Comparação dos processos propostos com outras abordagens.

\begin{tabular}{ccccccc}
\hline Complexidade & Processo I & Processo II & Agglomerative & DBSCAN & Spectral & K-Means \\
\hline \multirow{2}{*}{ Baixa } & $\mathbf{1 0 0 , 0 0 \%}$ & $\mathbf{1 0 0 , 0 0 \%}$ & $75,00 \%$ & $\mathbf{1 0 0 , 0 0 \%}$ & $75,00 \%$ & $75,00 \%$ \\
& $\mathbf{1 0 0 , 0 0 \%}$ & $\mathbf{1 0 0 , 0 0 \%}$ & $83,33 \%$ & $\mathbf{1 0 0 , 0 0 \%}$ & $93,33 \%$ & $83,33 \%$ \\
& $100,00 \%$ & $66,67 \%$ & $16,67 \%$ & $33,33 \%$ & $100,00 \%$ & $16,67 \%$ \\
\hline \multirow{2}{*}{ Média } & $50,00 \%$ & $50,00 \%$ & $75,00 \%$ & $50,00 \%$ & $75,00 \%$ & $75,00 \%$ \\
& $\mathbf{1 0 0 , 0 0 \%}$ & $71,43 \%$ & $57,14 \%$ & $57,14 \%$ & $57,14 \%$ & $71,43 \%$ \\
& $\mathbf{8 5 , 7 1 \%}$ & $\mathbf{8 5 , 7 1 \%}$ & $57,14 \%$ & $57,14 \%$ & $57,14 \%$ & $50,00 \%$ \\
\hline \multirow{2}{*}{ Alta } & $\mathbf{8 5 , 7 1 \%}$ & $57,14 \%$ & $42,86 \%$ & $14,29 \%$ & $\mathbf{8 5 , 7 1 \%}$ & $42,86 \%$ \\
& $\mathbf{8 5 , 7 1 \%}$ & $\mathbf{8 5 , 7 1 \%}$ & $71,43 \%$ & $71,43 \%$ & $28,57 \%$ & $42,86 \%$ \\
\hline Resultados & $\mathbf{8 5 , 7 1 \%}$ & $78,57 \%$ & $35,71 \%$ & $35,71 \%$ & $21,43 \%$ & $50,00 \%$ \\
\hline (Média) & $\mathbf{8 8 , 1 0 \%}$ & $77,25 \%$ & $57,14 \%$ & $57,67 \%$ & $65,93 \%$ & $56,35 \%$ \\
\hline
\end{tabular}

na nuvem de pontos, apresentam uma relevância maior em comparação com a cor. Observando os resultados, podemos concluir que a coloração dos objetos se utilizada como um peso ao invés de ser a principal informação norteadora da segmentação, talvez obtivesse melhores resultados.

Vale ressaltar que um ambiente real a disposição dos objetos estranhos estaria bem diferente das cenas de média e alta complexidade. As cenas que mais se aproximariam seriam as de complexidade baixa, visto que na maioria das vezes o objeto estranho é encontrado sozinho na pista.

\section{CONCLUSÃO}

Vimos que o problema de detritos em pistas de voo tem causado grandes prejuízos à comunidade da aviação civil e militar. O alto valor dispendido para manutenção gira em torno de bilhões de dólares ao ano. Os principais gastos diretos são para o concerto das turbinas dos aviões e os indiretos são difíceis de calcular pois repercutem por exemplo em atrasos de voos, combustível para aeronaves e despesas de hotéis para passageiros. Sem contar com os acidentes catastróficos já causados por detritos, levando a vida várias pessoas.

Dada a importância deste problema, diversas formas de mitigá-lo são estudadas. Limpeza e patrulhas manuais, utilização de carros limpadores, sensores micro-ondas presentes ao longo das pistas mostrando a localização exata dos detritos, todas essas abordagens já fazem parte do diaa-dia dos aeródromos. Observando esse problema, surge a oportunidade de empregar as tecnologias mais recentes relacionadas a locomoção autônoma e manipulação robótica para solução desta tarefa. Com o avanço da tecnologia em carros autônomos, é uma questão de tempo que soluções envolvendo tais tecnologias sejam utilizadas como produto ou serviço à disposição do aeroportos.

O presente trabalho vislumbra essa ideia e se posiciona primeiramente propondo atacar um problema bem específico que por isso só é um grande desafio: o problema da segmentação de nuvem de pontos. Estudando o comportamento dos detritos podemos constatar, que em um primeiro momento é mais interessante saber se tem algum objeto na pista do que identificá-lo ou classificá-lo, sem se importar, neste momento, com o quê o objeto é mas sim saber de sua existência no lugar errado. Partindo deste princípio adotamos sensores que entregam tal informação e nos concentramos na tarefa de segregar esses dados brutos no formato de nuvem de pontos.

Neste contexto, este trabalho propôs duas abordagens para fazer segmentação da nuvem de pontos, uma utilizando um método baseado em regiões e outra utilizando um método híbrido. Vimos que as abordagens apresentam resultados promissores e que valem a pena serem investigadas mais profundamente. Visto que propomos pré-processamentos que preparam o ambiente para que o processamento principal possa obter resultados melhores.

Este trabalho de forma alguma esgotou as possibilidades relacionadas à segmentação de nuvem de pontos. Trouxemos apenas uma nova perspectiva para aprimorar os resultados aplicando pré-processamentos no processo de detecção de objetos. Listando atividades que podem ser implementadas como extensão do presente trabalho, podemos citar:

- Benchmark com outros métodos: neste trabalho utilizamos apenas quatro algoritmos dos quais consideramos úteis e representativos de cada classe dos algoritmos de segmentação para nuvem de pontos.

- Utilização de novas métricas de avaliação: no contexto de métodos de segmentação de nuvem de pontos, existem várias métricas que se atentam para outras características. Métricas que avaliam tanto a qualidade quanto a quantidade da segmentação.

- Utilização de novos datasets: neste trabalho nos dispomos a utilizar o dataset $O S D$, devido a suas características de ter vários objetos dispostos no plano. Tal configuração serviu como simulação do que se pretende realizar em campo.

Em trabalhos futuros, a etapa de segmentação de nuvem de pontos fará parte de um processo completo de remoção de detritos. O processo completo ainda exigiria a investigação de métodos de manipulação robótica (planejamento de grasping), locomoção e navegação autônoma do robô e detecção de objetos por múltiplos sensores. Deste modo é possível construir o sistema completo para detecção e remoção de detritos ao longo das pistas de voo.

\section{REFERENNCIAS}

Andreas, R. (2015). The object segmentation database (osd). URL https://www.acin.tuwien.ac.at/ vision-for-robotics/software-tools/osd/.

Boeing (2018). Boeing. URL http://www.boeing.com/ commercial/aeromagazine/aero_01/s/s01/index. html.

Boularias, A., Bagnell, J.A., and Stentz, A. (2015). Learning to manipulate unknown objects in clutter by reinforcement. In Proceedings of the Twenty-Ninth AAAI Conference on Artificial Intelligence, 1336-1342. AAAI Press.

Castro, M. (2015). kmeans. URL https://github.com/ marcoscastro/kmeans.

FAA (2013). Faa. URL https://www.faa.gov/news/ fact_sheets/news_story.cfm?newsId=15394.

Fischler, M.A. and Bolles, R.C. (1981). Random sample consensus: a paradigm for model fitting with applications to image analysis and automated cartography. Communications of the ACM, 24(6), 381-395. 
Kim, D.I. and Sukhatme, G.S. (2014). Semantic labeling of $3 \mathrm{~d}$ point clouds with object affordance for robot manipulation. In Robotics and Automation (ICRA), 2014 IEEE International Conference on, 5578-5584. Citeseer.

Rusu, R.B. and Cousins, S. (2011). 3d is here: Point cloud library (pcl). In Robotics and automation (ICRA), 2011 IEEE International Conference on, 1-4. IEEE.

Ückermann, A., Haschke, R., and Ritter, H. (2012). Realtime $3 \mathrm{~d}$ segmentation of cluttered scenes for robot grasping. In Humanoid Robots (Humanoids), 2012 12th IEEE-RAS International Conference on, 198-203. IEEE.

UDT (2010). Advisory circular. URL https://www . skybrary.aero/bookshelf/books/3690.pdf.

Zrira, N., Ouadiay, F.Z., Hannat, M., Bouyakhf, E.H., and Himmi, M.M. (2017). Evaluation of pcl's descriptors for $3 \mathrm{~d}$ object recognition in cluttered scene. In Proceedings of the 2nd international Conference on Big Data, Cloud and Applications, 81. ACM. 